\title{
Innovation Qualité 2018 - die Gewinner (1)
}

\author{
Michelle Gerber ${ }^{a}$, Fabienne Hohl ${ }^{b}$ \\ a lic. phil. hum., wissenschaftliche Mitarbeiterin FMH, Abteilung Daten, Demographie und Qualität DDQ / Schweizerische Akademie für Qualität
} in der Medizin SAQM; ${ }^{\text {b }}$ Abteilung Kommunikation der FMH

Am 17. April 2018 wurde zum ersten Mal die Innovation Qualité verliehen, der neue Qualitätspreis der Schweizerischen Akademie für Qualität in der Medizin SAQM der FMH. Die Preisverleihung und die Präsentation der vier Gewinnerprojekte fanden am 1. SAQM-Symposium zum Thema «innovativ und translational» statt.

Die Innovation Qualité prämiert alle zwei Jahre Qualitätsprojekte aus dem Schweizer Gesundheitswesen, die sich in der Praxis bewährt haben. Sie macht damit Erfolge von Vordenkerinnen und Vordenkern der medizinischen Qualität aus verschiedensten Disziplinen und Berufen sichtbar und verschafft der Fachdiskussion zukunftsweisende Impulse. Die Innovation Qualité 2018 ist breit abgestützt: Sie wird von 23 ideellen Partnerorganisationen (siehe Kasten) getragen.

Bei 37 eingereichten Qualitätsprojekten hatten die beiden Jurys der Innovation Qualité keine leichte Aufgabe bei der Wahl der Gewinner. Eine Jury war für die Preiskategorie Patientenversorgung neu gedacht (Themenschwerpunkt 2018) sowie Ärzteorganisationen zuständig. Die andere Jury betreute die Kategorie Patientensicherheit in Zusammenarbeit mit Patientensicherheit Schweiz. $\mathrm{Zu}$ vergeben waren insgesamt 40000 Franken.

Die Preisverleihung der Innovation Qualité fand am 17. April 2018 im Rahmen des erstmalig durchgeführten SAQM-Symposiums zum Thema "innovativ und translational» statt. Zu Beginn der Veranstaltung gewährte Prof. Dr. med. em. Felix Frey interessante Einblicke in «Chancen und Nutzen der Translationalen Medizin für den Patienten». Der Geschäftsführer des Swiss Institute for Translational and Entrepreneurial Medicine (sitem-insel AG) erläuterte, wie aus Forschung und Entwicklung innovative Produkte und Instrumente zum Nutzen der Patienten entstehen. Der Hauptteil des Symposiums war der Preisverleihung der innovativen Qualitätsprojekte gewidmet: Den Gewinnern wurden feierlich ihre Diplome übergeben, und sie stellten ihre Projekte dem interessierten Fachpublikum vor.

Wir porträtieren die Gewinnerprojekte «Power-Patienten durch Chronic Care Management» der Kategorie
Patientenversorgung neu gedacht und "Aktive elektronische Überwachung der unerwünschten Arzneimittelwirkungen bei hospitalisierten Patienten" der Kategorie Patientensicherheit anschliessend in dieser Ausgabe der Schweizerischen Ärztezeitung. In der Kategorie Ärzteorganisationen entschied die Jury, den Preis gemeinsam an die beiden Projekte «Kampagne smarter medicine - Choosing Wisely Switzerland» und «Zertifikat Swiss Cancer Network» zu vergeben. Diese beiden Gewinner stellen wir in der kommenden Ausgabe 19/2O vor. Die SAQM der FMH gratuliert den vier Gewinnerteams ganz herzlich!

Informationen zur Innovation Qualité, zum SAQMSymposium sowie die Projektbeschriebe der Gewinner- und weiterer Projekte, welche in der engeren Auswahl der Jurys standen, finden Sie im Internet auf www.innovationqualite.ch

\section{Ideelle Partner}

\section{der Innovation Qualité 2018}

Bundesamt für Gesundheit BAG • curafutura • EQUAM Stiftung - Ergotherapeutlnnen-Verband Schweiz EVS • Faculté de biologie et de médecine de l'Université de Lausanne • Faculté de médecine de I'Université de Genève • Föderation Schweizer Psychologinnen und Psychologen FSP $\bullet \mathrm{H}+$ Die Spitäler der Schweiz - Insel Gruppe AG • Medizinische Fakultät der Universität Bern • Nationaler Verein für Qualitätsentwicklung in Spitälern und Kliniken ANQ • Patientenschutz SPO • pharmaSuisse Schweizerischer Apothekerverband • physioswiss • Schweizer Berufsverband der Pflegefachfrauen und Pflegefachmänner SBK • Schweizerische Akademie der Medizinischen Wissenschaften SAMW • Schweizerische Konferenz der kantonalen Gesundheitsdirektorinnen und -direktoren GDK • Schweizerischer Hebammenverband SHV • Schweizerischer Verband der Berufsorganisationen im Gesundheitswesen SVBG • Schweizerischer Verband der Ernährungsberater/innen SVDE • Schweizerischer Verband der Medizinischen Praxis-Fachpersonen SVA • Spitex Schweiz - UniversitätsSpital Zürich 


\title{
Power-Patienten dank interprofessionellem Chronic Care Management
}

\author{
Dr. med. Adrian Goeldlina, Dr. med. Marc Jungi ${ }^{a}$, Dr. med. Rahel Sahlia \\ a Sanacare $A G$
}

Demografische Veränderungen und Verknappung der Ressourcen prägen das Schweizer Gesundheitswesen $[1,2]$, und es wird Kritik laut an der Betreuungsqualität von chronisch Kranken [3, 4]. Als neuer Behandlungsstandard setzt sich die interprofessionelle Betreuung mit Fokus auf der Stärkung des Patienten-Selbstmanagements durch, z.B. beim Diabetes mellitus Typ 2 [5]. Mit dem strukturierten, interprofessionellen Chronic Care Management (CCM), das auf dem Modell von Wagner [6] beruht, hat sich die Sanacare AG dieser Herausforderungen erfolgreich angenommen.

In zwölf hausärztlichen Gruppenpraxen betreuen wir im CCM Patientinnen und Patienten mit Bluthochdruck, Diabetes mellitus Typ 2 und chronischer obstruktiver Lungenkrankheit (COPD). Das interprofessionelle Behandlungsteam besteht aus Ärztin oder Arzt und Coach (Medizinische Praxiskoordinatorin klini-

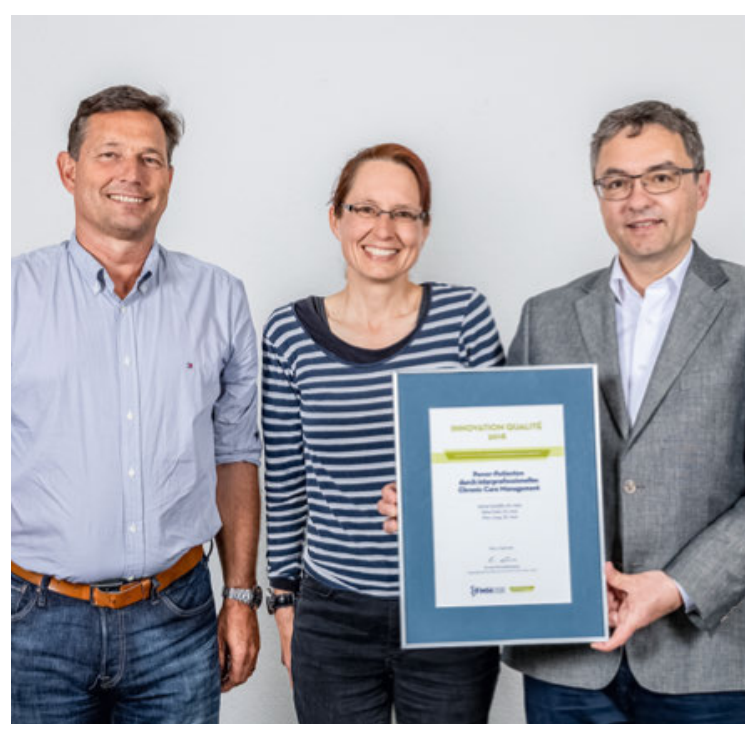

Das Gewinnerteam in der Kategorie «Patientenversorgung neu gedacht»: Marc Jungi, Rahel Sahli und Adrian Göldlin.

\begin{tabular}{|c|c|c|c|}
\hline \multirow[t]{2}{*}{ Anz. abgeschlossener Zyklen: } & \multicolumn{3}{|c|}{221} \\
\hline & Beginn & Ende & Veränderung \\
\hline Body-Mass-Index (im Ø) & 31.7 & 31.1 & -0.6 \\
\hline Gewicht (Kg im Ø) & 89.4 & 87.1 & -2.3 \\
\hline \multicolumn{4}{|l|}{ LDL-Cholesterin } \\
\hline $\mathrm{LDL}(\mathrm{mmol} / \mathrm{l} \mathrm{im} \varnothing)$ & 2.7 & 2.7 & -0.1 \\
\hline $\mathrm{LDL}<3 \mathrm{mmol} / \mathrm{l}$ in \% & $64 \%$ & $63 \%$ & $-1 \%$ \\
\hline LDL $3.00-3.49 \mathrm{mmol} / \mathrm{in} \%$ & $15 \%$ & $16 \%$ & $1 \%$ \\
\hline $\mathrm{LDL} 3.50-3.99 \mathrm{mmol} / \mathrm{l}$ in $\%$ & $8 \%$ & $9 \%$ & $1 \%$ \\
\hline $\mathrm{LDL} \geq 4.00 \mathrm{mmol} / \mathrm{in} \%$ & $14 \%$ & $12 \%$ & $-1 \%$ \\
\hline \multicolumn{4}{|l|}{ Blutdruckwerte } \\
\hline Blutdruck systolisch (im Ø) & 142 & 137 & -6 \\
\hline Blutdruck diastolisch (im Ø) & 82 & 79 & -3 \\
\hline Blutdruck normal in \% & $41 \%$ & $56 \%$ & $15 \%$ \\
\hline Bluthochdruck Grad 1 in \% & $39 \%$ & $35 \%$ & $-4 \%$ \\
\hline Bluthochdruck Grad 2 in \% & $18 \%$ & $8 \%$ & $-11 \%$ \\
\hline Bluthochdruck Grad 3 in \% & $2 \%$ & $1 \%$ & $-1 \%$ \\
\hline \multicolumn{4}{|c|}{ Marker für die Blutzuckereinstellung } \\
\hline $\mathrm{HbA} 1 \mathrm{c}(\mathrm{im} \varnothing)$ & 7.2 & 6.7 & -0.5 \\
\hline $\mathrm{HbA} 1 \mathrm{c}<6.5 \mathrm{mmol} / \mathrm{l}$ in \% & $31 \%$ & $44 \%$ & $13 \%$ \\
\hline $\mathrm{HbA} 1 \mathrm{c} 6.5$ bis $7.49 \mathrm{mmol} / \mathrm{l}$ in \% & $41 \%$ & $44 \%$ & $3 \%$ \\
\hline $\mathrm{HbA} 1 \mathrm{c} 7.5$ bis $8.49 \mathrm{mmol} / \mathrm{l}$ in \% & $17 \%$ & $8 \%$ & $-9 \%$ \\
\hline $\mathrm{HbA} 1 \mathrm{c} \geq 8.5 \mathrm{mmol} / \mathrm{l}$ in $\%$ & $11 \%$ & $4 \%$ & $-7 \%$ \\
\hline \multicolumn{4}{|l|}{ Körperliche Aktivität } \\
\hline Aktivität 3 (aktiv) & $23 \%$ & $33 \%$ & $9 \%$ \\
\hline Aktivität 2 (gering aktiv) & $36 \%$ & $39 \%$ & $3 \%$ \\
\hline Aktivität 1 (inaktiv) & $41 \%$ & $29 \%$ & $-12 \%$ \\
\hline
\end{tabular}

Tabelle 1: Veränderung von klinischen Outcomes nach 221 ersten Zyklen CCM (Empowerment) bei Patientinnen und Patienten mit Diabetes mellitus Typ 2 und mit gleichzeitiger arterieller Hypertonie. Die Zahlen sind gerundet. scher Richtung oder fortgeschritten in der Ausbildung dazu). Das CCM läuft in Jahreszyklen ab. Gemeinsam mit den Patienten werden für jeden Programmzyklus Ziele schriftlich festgehalten und unterschrieben. Klar definierte und elektronisch unterstützte Behandlungspfade garantieren eine guidelinegerechte Betreuung. Unser CCM besteht aus zwei Phasen:

1. Empowerment: Diese Phase befähigt die Patienten zum Selbstmanagement: Sie erarbeiten grundlegendes Wissen über ihre Krankheit, Strategien zum Umgang damit sowie konkrete Anpassungen ihres Lebensstils.

2. Erhaltung: Während dieser Phase wird etwa die Hälfte der notwendigen medizinischen Kontrollen an die Coaches delegiert.

Aktuell behandeln wir gegen 600 Menschen mit chronischen Erkrankungen im CCM. Tabelle 1 zeigt, dass das Empowerment (1. Zyklus) auch bei bereits zu Beginn recht gut behandelten Patienten noch Verbesserungen bringt. Die Betreuungsqualität der Typ-2Diabetiker in unserem CCM ist gemäss den Qualitätsindikatoren der Schweizerischen Gesellschaft für 
Korrespondenz: Sanacare Gruppenpraxis Bern Bubenbergplatz 10 CH-3011 Bern Tel. 0313857111 adrian.goeldlin[at] sanacare.ch
Endokrinologie und Diabetologie hoch. Ebenfalls hoch ist die Patientenzufriedenheit.

Unser Hauptbestreben in den nächsten Jahren wird sein, möglichst viele Patientinnen und Patienten mit den genannten Diagnosen im Rahmen eines CCM zu betreuen. Wir sind überzeugt, dass sich ein solches Chronic-Care-Management-System auch in weiteren Hausarztpraxen implementieren lässt. Mittelfristig möchten wir weitere Versorgungspartner (verschiedene Gesundheitsberufe) einbinden. Auch die Patientensicht soll künftig vermehrt in die Entwicklung einfliessen.

\section{Literatur}

1 Schweiz. Gesundheitsobservatorium (Hrsg.). Gesundheit in der Schweiz - Fokus chronische Erkrankungen. Nationaler Gesundheitsbericht 2015. Bern: Hogrefe; 2015.

2 Zeller A, Tschudi P. Erhebung der Workforce Hausarztmedizin in der Schweiz 2015. Primary and Hospital Care. 2016;16(15):277-80.

3 Huber CA, et al. A set of four simple performance measures reflecting adherence to guidelines predicts hospitalization: a claimsbased cohort study of patients with diabetes. Patient Preference and adherence. 2016;10:223-31.

4 Walther D, et al. High blood pressure: prevalence and adherence to guidelines in a population-based cohort. Swiss Med Wkly. 2016;146:w14323.

5 Beck J, et al. 2017 National Standards for Diabetes Self-Management Education and Support. Diabetes Care. 2017;40:1409-19.

6 Wagner EH. Chronic disease management: what will it take to improve care for chronic illness? Eff Clin Pract. 1998;1:2-4.

\title{
Elektronische Überwachung unerwünschter Arzneimittelwirkungen
}

\author{
Alessandro Ceschi, PD Dr. med.a, b; Paolo Hitz, MMeda; Laura Müller, Dipl. Pharm.a; \\ Gerd Kullak-Ublick, Prof. Dr. med. ${ }^{\mathrm{b}}$; Vasco Piffaretti, BSc ${ }^{\mathrm{c}}$
}

${ }^{a}$ Clinica di farmacologia e tossicologia clinica, Istituto di Scienze Farmacologiche della Svizzera Italiana, Ente Ospedaliero Cantonale, Lugano; ${ }^{b}$ Klinik für klinische Pharmakologie und Toxikologie, UniversitätsSpital Zürich; ${ }^{c}$ Area Information and Communications Technology, Ente Ospedaliero Cantonale, Bellinzona

Die Marktzulassung für ein Arzneimittel bedeutet keine absolute Sicherheit des Medikaments für die Patientin, den Patienten. Das Bundesgesetz über Arzneimittel und Medizinprodukte (Heilmittelgesetz) sieht die Meldepflicht für unerwünschte Arzneimittelwirkungen (UAW) durch medizinische Fachpersonen vor. Die Spontanmeldung von UAW oder UAW-verdächtigen Ereignissen bildet weltweit die Grundlage der Pharmakovigilanz. Ihr Hauptschwachpunkt ist das Nicht-Melden von unter Umständen relevanten UAW. Dieses Underreporting wird bis auf $94 \%$ geschätzt. Das Ziel des Projekts war, ein aktives, einfaches elektronisches Pharmakovigilanz-System bei hospitalisierten Patienten zu entwickeln und zu testen. Dieses System sollte sowohl UAW, die vor dem Eintritt ins Spital entstanden sind und zur Hospitalisation geführt oder beigetragen haben, als auch UAW während der Hospitalisation erfassen.

\section{Die richtigen Fachtermini filtern}

In enger Zusammenarbeit mit der ICT des Spitalnetzes Ente Ospedaliero Cantonale (EOC) wurde ein elektro-

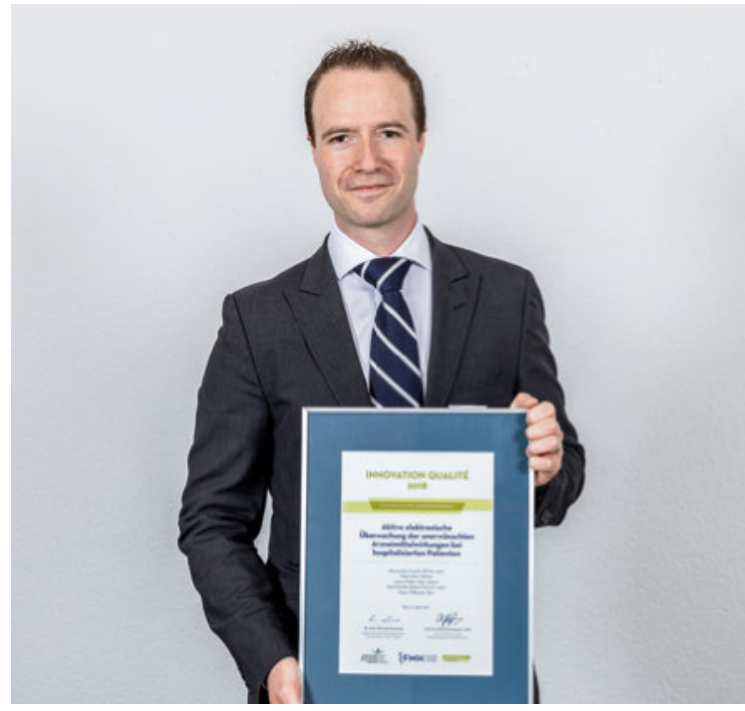

Das Gewinnerdiplom der Innovation Qualité in der Kategorie "Patientensicherheit» geht an Alessandro Ceschi.

nisches System entwickelt, welches alle Textspeicherprozesse in den - geschützten - digitalen Patientendossiers filtert. Dabei sucht es nach ausgewählten 
Ausdrücken, die auf UAW hinweisen. In der ersten Phase wurden alle Patientendossiers der vom System gemeldeten Fälle manuell detailliert analysiert und ausgewertet, um die Leistung des elektronischen Systems im Sinne der Spezifität der gemeldeten Fälle zu testen. Nach einigen Anpassungen der Fachwörterliste zeigt sich eine statistisch signifikante Verbesserung im Vergleich zur ersten Version ( $p=0,001$ ).

\section{Fälle in 51 Tagen}

Korrespondenz: PD Dr. med. Alessandro Ceschi, FEAPCCT

Farmacologia e tossicologia

clinica, Medicina interna

generale

Direttore medico e scienti-

fico, Istituto di Scienze Farmacologiche della Svizzera

Italiana Ente Ospedaliero

Cantonale

Via Tesserete 46

CH-6900 Lugano

Tel. 0918116863

Fax 0918116751
In der Beobachtungsperiode von insgesamt 51 Tagen wurden vom System 365 Fälle gemeldet. Davon waren $77,5 \%(n=283)$ echte UAW und 56,5\% ( $\mathrm{n}=160)$ meldepflichtige UAW. Von letzteren waren $87,5 \%(n=140)$ schwerwiegende UAW, 11,9\% ( $\mathrm{n}=19)$ medizinisch wichtige UAW und $0,6 \%(n=1)$ UAW aufgrund von Medikamentenmissbrauch. Von den 140 schwerwiegenden UAW erforderten $60,7 \%(n=85)$ eine Hospitalisierung, die anderen 39,3\% $(n=55)$ verlängerten den Spitalaufenthalt. Von den 160 meldepflichtigen UAW wurden dem Pharmakovigilanz-Zentrum insgesamt nur 8,8\%
( $\mathrm{n}=14)$ spontan durch medizinische Fachpersonen gemeldet; die Underreporting-Rate betrug also 91,3\%.

\section{Underreporting erfolgreich eingedämmt}

Das Suchsystem wurde dann erfolgreich in die tägliche Arbeit des regionalen Pharmakovigilanz-Zentrums der italienischen Schweiz eingeführt und liefert heute einen wichtigen Beitrag zur Reduktion des Underreportings - und damit zur besseren Erfassung und Überwachung der UAW in der Region. Swissmedic hat diese Entwicklung positiv beurteilt. Nach Übersetzung der Ausdrücke in die anderen Landessprachen könnten auch die anderen regionalen PharmakovigilanzZentren dieses einfache Suchsystem verwenden. Was das Problem des Underreportings auf nationalem Niveau beträchtlich reduzieren könnte und damit zur weiteren Erhöhung der Arzneimittelsicherheit in der Schweiz beitrüge.

Bildnachweise

Fotos der Gewinner: FMH

Tabelle: Sanacare AG 